ARTICLE

\title{
A method for controlling the synthesis of stable twisted two-dimensional conjugated molecules
}

Yongjun $\mathrm{Li}^{1}$, Zhiyu Jia ${ }^{1}$, Shengqiang Xiao ${ }^{2}$, Huibiao Liu ${ }^{1} \&$ Yuliang $\mathrm{Li}^{1}$

Thermodynamic stabilization ( $\pi$-electron delocalization through effective conjugation) and kinetic stabilization (blocking the most-reactive sites) are important considerations when designing stable polycyclic aromatic hydrocarbons displaying tunable optoelectronic properties. Here, we demonstrate an efficient method for preparing a series of stable two-dimensional (2D) twisted dibenzoterrylene-acenes. We investigated their electronic structures and geometries in the ground state through various experiments assisted by calculations using density functional theory. We find that the length of the acene has a clear effect on the photophysical, electrochemical, and magnetic properties. These molecules exhibit tunable ground-state structures, in which a stable open-shell quintet tetraradical can be transferred to triplet diradicals. Such compounds are promising candidates for use in nonlinear optics, field effect transistors and organic spintronics; furthermore, they may enable broader applications of 2D small organic molecules in high-performance electronic and optical devices.

\footnotetext{
${ }^{1}$ CAS Key Laboratory of Organic Solids, Beijing National Laboratory for Molecular Science (BNLMS), Institute of Chemistry, Chinese Academy of Sciences, Beijing 100190, China. ${ }^{2}$ State Key Laboratory of Advanced Technology for Materials Synthesis and Processing, Wuhan University of Technology, Wuhan 430070, China. Correspondence and requests for materials should be addressed to Yongjun Li (email: liyj@iccas.ac.cn) or to Yuliang Li (email: ylli@iccas.ac.cn).
} 
$\mathrm{n}$ the last two decades, methodologies for the controlled synthesis of large $\pi$-extended polycyclic aromatic hydrocarbons (PAHs) have been established using bottom-up principles $^{1-11}$. The properties of PAHs depend heavily on their degrees of $\pi$-extension, shapes, widths and edge topologies $1,3,4,12-14$. Many linear acenes, including tetracene, pentacene and related derivatives, have been synthesized to produce highly desirable electronic properties, including remarkable charge-carrier mobilities $^{15-19}$. Open-shell PAHs, which feature one or more $\pi$-electrons not tightly paired into the bonding molecular orbital in the ground state, are particularly interestingly structures that have properties very different from those of typical PAHs having closedshell electronic structures ${ }^{20-23}$. The unique electronic structures of two-dimensional (2D) open-shell PAHs can impart attractive electronic, optical and magnetic properties for applications in materials science ${ }^{24-26}$.

Acenes become increasingly reactive as the number of rings increases, with the central ring being the most reactive ${ }^{27,28}$. As a result, the central rings in these molecules are susceptible to oxidation, photodegeneration and Diels-Alder reactions ${ }^{29,30}$. Open-shell molecules are particularly vulnerable to degradation reactions ${ }^{22,23}$; therefore, instability remains a key obstacle affecting their practical applications. In most cases, thermodynamic stabilization ( $\pi$-electron delocalization through effective conjugation) and kinetic stabilization (blocking of the most-reactive sites) are both necessary to obtain stable materials ${ }^{18,31}$. One strategy is to annulate the aromatic rings onto two neighbouring rings, creating $2 \mathrm{D}$ acene analogues ${ }^{15}$. Changing from the linear character of a condensed array (peri-condensed) to an angular geometry (cata-condensed) results in at least two sextets of $\pi$-electrons, increasing the stability relative to that of a linear analogue having only one sextet ${ }^{32}$. Tuning a planar structure to a nonplanar structure through twisting or saddling can also enhance the kinetic stability as a result of steric blocking 21,33 .

$2 \mathrm{D}$ acene analogues that represent various fragments of graphene have received significant attention because of their electronic properties ${ }^{6,9,10,15}$. Molecules of this class have large, planar $\pi$-surfaces allowing high-intermolecular surface overlap ${ }^{34-36}$. The packing of such molecules is dictated by multiple interactions that can effectively increase the dimensionality of the electronic structure, leading to enhanced transport properties ${ }^{37,38}$. Many 2D planar PAHs $11,32,34,36,39-41$ and contorted polycyclic aromatics ${ }^{5,42-44}$ have been synthesized and characterized recently.

In this paper, we report the transformation of a one-dimensional (1D) single conjugated dibenzoterrylene through the attachment of conjugated acene 'arms' to generate a 2D molecular structure. High dimensionality within the single molecule was assured by hybridizing dibenzoterrylene with acenes of different conjugation lengths; stability was provided by benzoannulating the active rings of acenes and inducing contortions from the cove or fjord regions. This protocol is applicable to construct various $2 \mathrm{D}$ twisted nanographene-like structures in a rapid manner.

\section{Results}

Design of target compounds. Figure 1a showed the conceptual approaches toward twisted 2D acenes. We positioned electrondonating/withdrawing groups (for example, $\mathrm{CH}_{3}, \mathrm{OMe}, \mathrm{CN}, \mathrm{F}$ ) to alter the optical absorbance and the energy levels of the highest occupied molecular orbital (HOMO) and lowest unoccupied molecular orbital (LUMO) of the tetracene-dibenzoterrylene hybrid, in addition to tuning the molecular packing in the solid state (Fig. 1b). We introduced phenanthrenyl groups to further increase the steric constraint, and naphthyl and perylenyl groups to elongate the acenes and allow annulation of benzene rings. Using this approach, 2D twisted acenes varying from closed-shell to open-shell structures were available through extension of the acenes vertical to the benzoterrylene and benzoannulation at the zigzag edge. Compound $\mathbf{4}$ can be considered (Fig. 1c) as a hybrid of two pentacene-annulated-tribenzo-octacenes, in which two phenalenyl radicals (5,10 positions of octacene) are annulated with a central tetracene unit, or having evolved from a dibenzopentacene with the reactive $5^{\prime}, 7^{\prime}$-positions of the pentacene connected and protected together. In both cases, one more Clar sextet was formed on resonance to tetraradical state, which also predicted the stability of the tetraradical.

Synthesis of target compounds. Figure 2 displays the synthesis of $\mathbf{1}$, starting from the bromination of the amine $\mathbf{5}$ (ref. 45) with $\mathrm{Br}_{2}$ followed by conversion of the amino group to the iodide through diazotization. Compound 7 was coupled with 2,7-bis $(4,4,5,5$ tetramethyl-1,3,2-dioxaborolan-2-yl)pyrene through SuzukiMiyaura cross-coupling to give the key intermediate 8. This compounds was coupled with a series of $\mathrm{RB}(\mathrm{OH})_{2}$ derivatives 9 to provide the precursors 10a-g for Scholl-type oxidative cyclodehydrogenation, in which $\mathrm{FeCl}_{3}$ was used as both a Lewis acid and an oxidant. tert-Butyl groups were introduced to increase the solubility of the final product. A phenanthrenyl group was used to further increase the steric constraint, leading to precursor 11; naphthyl and perylenyl groups were introduced to elongate the acenes and introduce the annulation benzene rings, providing the precursors 12 and 13, respectively.

For 10e, $\mathrm{C}-\mathrm{C}$ bonds were formed ortho and para to the meta fluorine atom, leading to a mixture where the fluorine atoms were located at the $\mathrm{R}_{1}$ and $\mathrm{R}_{3}$ positions, as supported in the $\mathrm{X}$-ray structure (see below). For 11, the Scholl reaction at the K-region (convex armchair edge) of the phenanthrenyl group led to 2 in $57 \%$, which is too crowded at the fjord regions to allow two benzene rings to get sufficiently close to form a further $\mathrm{C}-\mathrm{C}$ bond $^{46}$. $\pi$-Conjugation extended compound 3 was obtained from 12 in $78 \%$ yield. The oxidative cyclohydrogenation of 13 is timedependent. When the reaction time was $<2 \mathrm{~h}$, we obtained some incompletely cyclized products, which were difficult to separate from the target 4 ; when the reaction time was longer than $12 \mathrm{~h}$ we obtained some byproducts with similar molecular weight as 4 but with different absorption spectra. The black compound 4 is wellsoluble in $\mathrm{C}_{2} \mathrm{H}_{2} \mathrm{Cl}_{4}$ and $o$-dichlorobenzene (dark-green solution), partially soluble in $\mathrm{CH}_{2} \mathrm{Cl}_{2}$ and $\mathrm{CHCl}_{3}$, we could purify it (in $51 \%$ yield) through column chromatography on deactivated silica gel with $\mathrm{CH}_{2} \mathrm{Cl}_{2} / \mathrm{MeOH} \quad(5 / 1)$. We characterized all of the intermediates and final products, except for 4, using ${ }^{1} \mathrm{H}$ and ${ }^{13} \mathrm{C}$ nuclear magnetic resonance (NMR) spectroscopy and highresolution mass spectrometry (MS); we characterized compound 4 only through high-performance liquid chromatography, highresolution MS and elemental analysis.

Conformations characterization of compounds 1-3. At first, we performed density functional theory (DFT) optimization to gain insight into the molecular conformations of these $2 \mathrm{D}$ acenes. The nonplanar conformations of these compounds arose from severe steric strain between the two $\mathrm{C}-\mathrm{H}$ bonds at the cove or fjord regions, as can be seen clearly in the model of $\mathbf{3}$ in Fig. 3a. DFT calculations revealed that $\mathbf{3}$ has five possible conformations (Fig. 3a); the hexacene units can take on conformations that are twisted or anti-folded with the central naphthalene unit, while the outer naphthalene rings in the hexacenes can be contorted out of the plane defined by the dibenzoterrylene core in the cis or trans direction: $c \mathrm{AA}-\mathbf{3}, t \mathrm{AA}-3, \mathrm{AT}-\mathbf{3}, c \mathrm{TT}-\mathbf{3}$ and $t \mathrm{TT}-\mathbf{3}$. Compound 1 

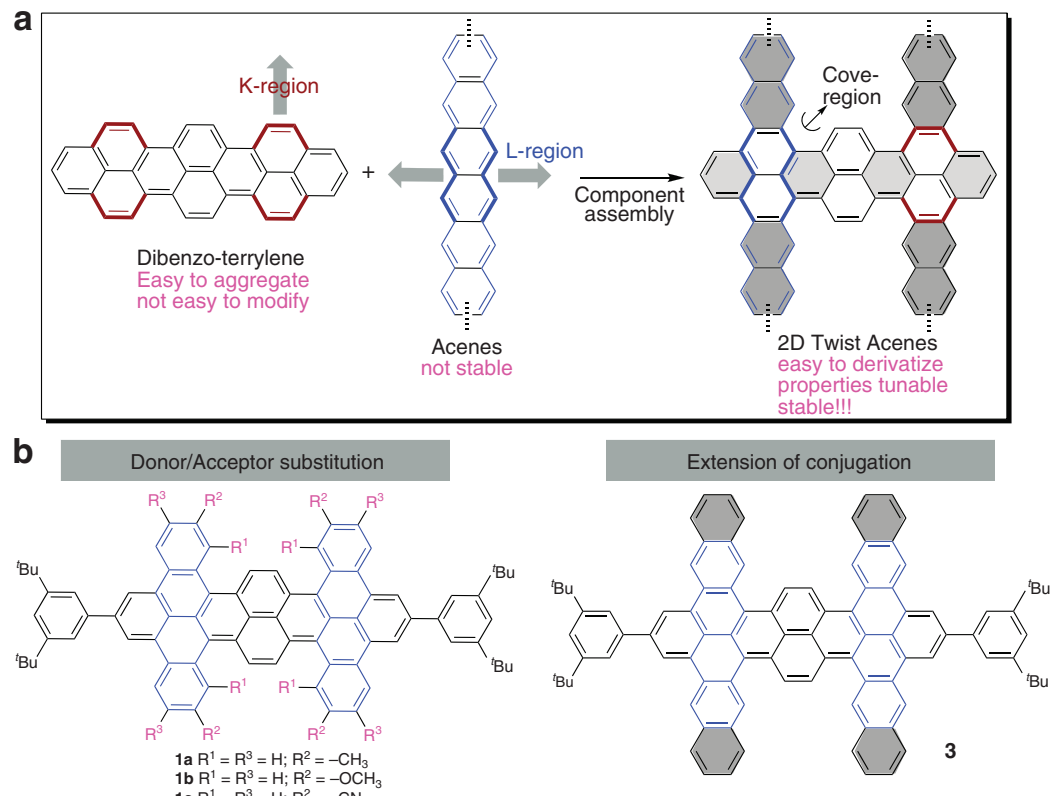
$1 c R^{1}=R^{3}=H ; R^{2}=-C N$
$1 d R^{1}=R^{3}=H ; R^{2}=-F$

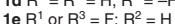

1f $R^{1}$ or $R^{3}=F \cdot R^{2}=F$

$1 g R^{1}=R^{2}=R^{3}=-F$

Extension of conjugation Benzoannulation
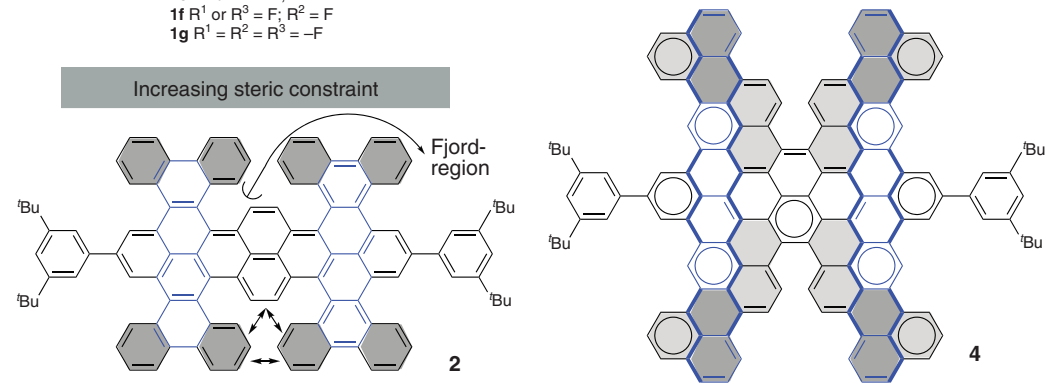

C
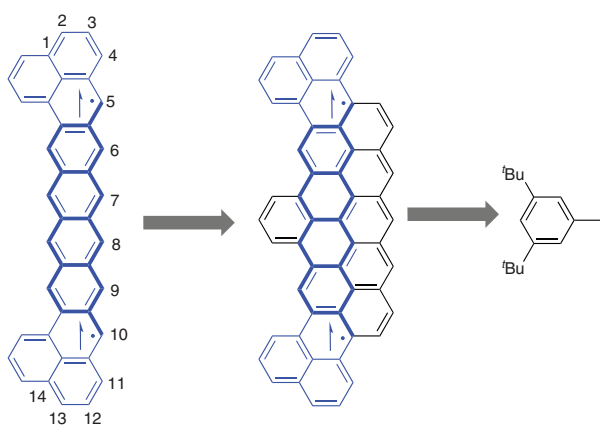

dibenzooctacene (DBO)

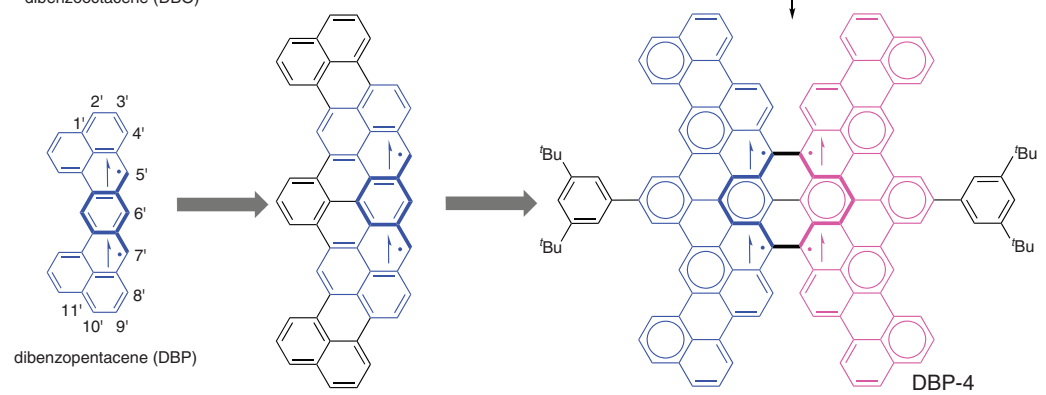

Figure 1 | Conceptual approaches toward twisted 2D acenes. (a) $\pi$-Components of 2D acenes. (b) Molecular structures of 2D acenes with increasing steric constraint (2), extension of conjugation (3) and with both extension of conjugation and benzoannulation at the L-region (zigzag edge; 4).

(c) Chemical structural evolution of 4: (top) Benzoannulation of octacene to generate phenalenyl radicals annulated with a central tetracene and further pentacene-annulation on the other side to provide more stable diradicals, two of which connect in the central area; (bottom) evolution from a dibenzopentacene with benzo- and naphtheno-annulation, with the reactive $5^{\prime}, 7^{\prime}$-positions of the pentacene connected and protected together. 


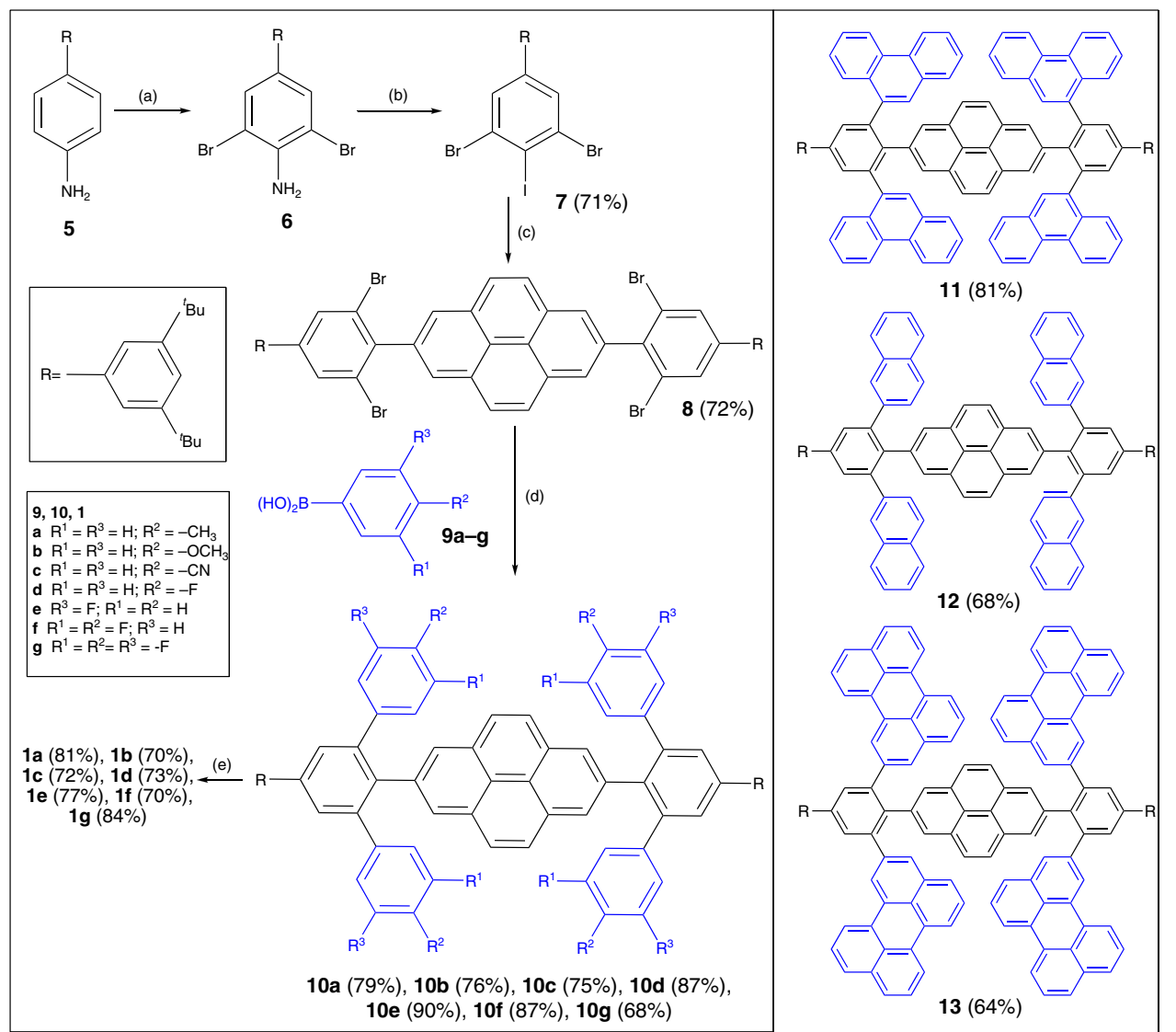

Figure 2 | Synthesis of the tetracene-dibenzoterrylene hybrids 1a-f and the precursors 11-13 for generation of 2-4. (a) $\mathrm{Br}_{2}, \mathrm{DCM} / \mathrm{MeOH}$; (b) $\mathrm{NaNO}{ }_{2}$, $\mathrm{AcOH} / \mathrm{H}_{2} \mathrm{SO}_{4}, \mathrm{KI} / \mathrm{I}_{2} ;$ (c) 2,7-bis (4,4,5,5-tetramethyl-1,3,2-dioxaborolan-2-yl)pyrene, $\mathrm{Pd}\left(\mathrm{PPh}_{3}\right)_{4}, \mathrm{Na}_{2} \mathrm{CO}_{3}, \mathrm{THF} / \mathrm{H}_{2} \mathrm{O}$; (d) $\mathrm{Pd}(\mathrm{PPh})_{4}, \mathrm{Na} 2 \mathrm{CO}$, toluene/ $\mathrm{EtOH} / \mathrm{H}_{2} \mathrm{O}$; (e) $\mathrm{FeCl}_{3}, \mathrm{CH}_{3} \mathrm{NO}_{2}, \mathrm{CH}_{2} \mathrm{Cl}_{2}, 4 \mathrm{~h}$.

existed in conformations analogous to those of $\mathbf{3}$ (Supplementary Fig. 69; Fig. 3 b displays the most stable conformation of 1a). In all of these cases, the $\mathrm{C}_{2}$-symmetric $c \mathrm{AA}$ structures were calculated to be more stable than the other conformers by $3.03-24.65 \mathrm{~kJ} \mathrm{~mol}^{-1}$ (Supplementary Table 1).

We grew single crystals through diffusion of acetone into a solution of $1 \mathbf{e}$ in $\mathrm{CHCl}_{3}$ (Supplementary Table 2). Figure $3 \mathrm{~d}$ displays the crystal structure of 1e. The fluorinated outer aromatic rings are contorted, in the same direction, out of the plane defined by the dibenzoterrylene core, resulting in a saddlelike conformation, consistent with the calculated gas-phase structure $(c \mathrm{AA}-1 \mathrm{e})$. The degree of twisting $(\theta)$, measured as the dihedral angle between the central naphthalene and the fluorinated outer benzene/terminal benzo group, were in the range of $32.2-37.3^{\circ}$ (Fig. 3e). Molecules of 1e were stacked in columns along the $c$-axis (Fig. $3 \mathrm{f}$ ). Within a columnar stack, two saddle molecules with curvature in the same direction formed a $\pi$-dimer with effective $\pi-\pi$ surface overlap; this $\pi$-dimer then stacked with another $\pi$-dimer of opposite molecular orientation.

For compound 2, we predicted a similar set of five possible conformations (Supplementary Fig. 70), with $c$ TT-2 calculated as the most stable because of additional steric strain between the phenanthrenyl moieties (Fig. 3c). Fortunately, this zero-dipolemoment conformer $c$ TT- 2 could be separated through column chromatography. Through slow diffusion of acetone into a solution of $c$ TT-2 in $\mathrm{CHCl}_{3}$, were obtained crystals for X-ray analysis, although they diffracted only weakly (Supplementary Fig. 71). A partial solution revealed that the phenanthrenyl units were twisted with respect to the central naphthalene unit in the cis direction. Although frozen in a single conformation as a result of crystal packing constraints in the solid state, these $2 \mathrm{D}$ acenes are highly flexible in solution and change their shape constantly on heating (Supplementary Fig. 72).

Optical and electrochemical properties of compounds 1-3. We measured the ultraviolet-visible absorption spectra of compounds 1-3 in $\mathrm{CH}_{2} \mathrm{Cl}_{2}$ solution (Fig. 4a-d, Supplementary Fig. 73, Supplementary Table 3). Absorption bands appeared in the ranges $300-400 \mathrm{~nm}$ and $400-600 \mathrm{~nm}$ with molar absorptivities of $>50,000 \mathrm{M}^{-1}$. The substituents and lengths of the acenes appeared to influence the $\pi$-stacking of these $2 \mathrm{D}$ acenes. For 1 , three peaks in the range $400-600 \mathrm{~nm}$, attributed to $0 \rightarrow 0,0 \rightarrow 1$ and $0 \rightarrow 2$ vibrational absorptions, were good handles for observation of its aggregation. With the increasing of concentration, the intensity of the $0 \rightarrow 0$ absorption decreased; in particular, the $0 \rightarrow 0$ absorption became a shoulder for the CN-substituted 1c, even disappearing for the trifluorine-substituted $1 \mathrm{~g}$. These results suggested the permanent formation of $\mathrm{H}$-aggregated $\pi$-dimers, as in the crystal structure of 1e (Fig. 3d) and the molecular modelling of $\mathbf{1 g}$ (Fig. 3g), in solution. On increasing the concentration, the intensity of the $0 \rightarrow 0$ vibrational absorption decreased further (Supplementary Fig. 74), with the $0 \rightarrow 2$ vibrational absorptions becoming the main absorption bands in the spectra of spin-coated thin film of $\mathbf{1 c - f}$, consistent with further $\mathrm{H}$-aggregation of their $\pi$-dimers. In the thin films of $\mathbf{1 a}$ and $\mathbf{1 b}$, in addition to $\mathrm{H}$-aggregation we also observed some J-aggregates, as indicated by the red-shifting of new bands. Such J-aggregates were also found for compounds 2 and $\mathbf{3}$ with bulky acenes, with red-shifting of the absorption maxima $\left(\lambda_{\max }\right)$. 
a

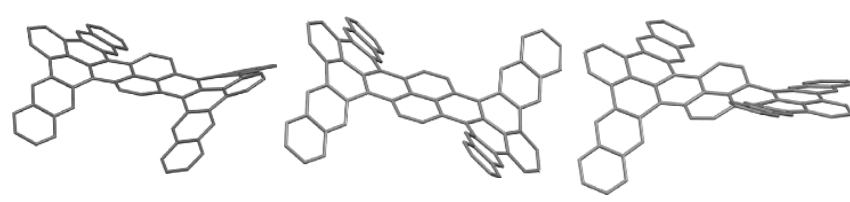

$s A A$

tAA

AT

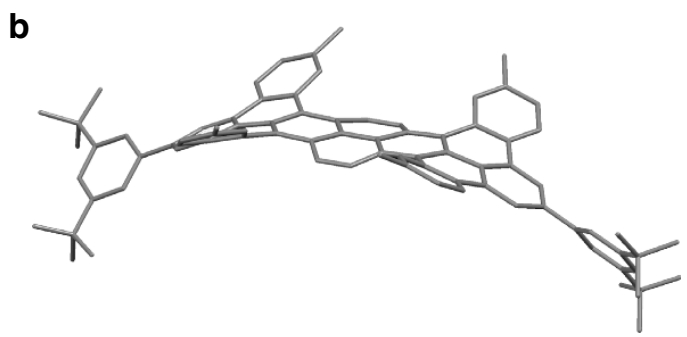

d

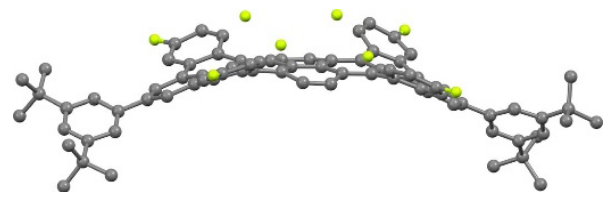

e

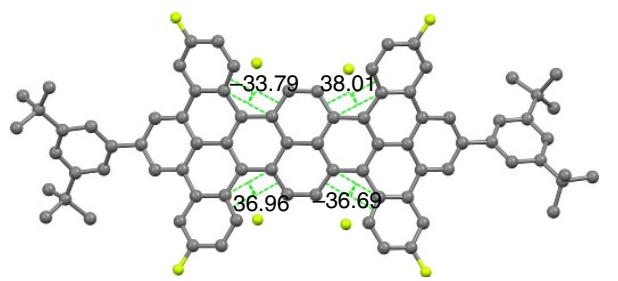

C
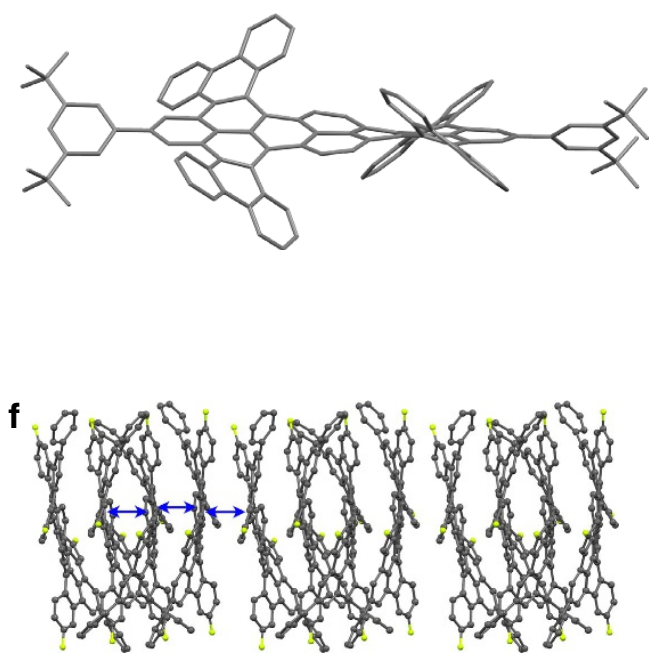

Figure $\mathbf{3}$ | Conformational flexibility of the 2D acenes. (a) Possible molecular conformations of $\mathbf{3}$ and (b,c) the most stable conformations of (b) $\mathbf{1 a}$ and (c) 2, calculated at the B3LYP/6-31G (d) level of theory. cAA-: anti-folded and anti-folded in the cis direction; tAA-: anti-folded and anti-folded in the trans direction; AT-: anti-folded and twisted; cTT-: twisted and twisted in the cis direction; tTT-: twisted and twisted in the trans direction. (d) Side and (e) top views of the $\pi$-backbone of $\mathbf{1 e}$ from crystal structure analysis; (f) molecular packing of $\mathbf{1 e}$, viewed along the $c$-axis of the unit cell; the hydrogen atoms and tert-butyl groups have been omitted for clarity; carbon and fluorine atoms are coloured grey and green, respectively.

The photoluminescence features of these compounds in dilute solution $\left(10^{-3} \mathrm{mmoll}^{-1}\right)$ were complementary of their absorption spectra; quantum yields were in the range 0.52-0.89 (Supplementary Table 3). We observed large bathochromic emission features in the emission spectra of these compounds on concentrating or going from solution to the solid state, consistent with strong aggregation. For the tetracene-dibenzoterrylene 1, we observed a bathochromic emission shift from 108 to $190 \mathrm{~nm}$; the more bulky compounds 2 and $\mathbf{3}$ featured shifts of only 24 and $71 \mathrm{~nm}$, respectively.

We used cyclic voltammetry (CV) and differential pulse voltammetry to examine the electrochemical behaviour of 1-3 in $\mathrm{CH}_{2} \mathrm{Cl}_{2}$ containing $0.1 \mathrm{M} \mathrm{TBAPF}_{6}$ as the supporting electrolyte (Supplementary Fig. 75, Supplementary Table 3). The CV traces of $\mathbf{1 a}-\mathbf{c}, \mathbf{1 e}, \mathbf{1 f}$ and $\mathbf{2}$ each featured two well-defined, reversible oxidation potentials. The cyclic voltammogram of $\mathbf{3}$ was more remarkable, displaying three oxidation potentials for four electrons at $+0.25 \mathrm{~V}(\mathbf{1 e}),+0.51 \mathrm{~V}(\mathbf{1 e})$ and $+0.72 \mathrm{~V}(\mathbf{2 e})$, all as fully reversible waves (Fig. 4e). Compound 1d exhibited a similar oxidation behaviour, with signals at $+0.42 \mathrm{~V}(\mathbf{1 e})$, $+0.63 \mathrm{~V}(\mathbf{1 e})$ and $+0.87 \mathrm{~V}$ (2e) (versus $\mathrm{Fc}_{\mathrm{Fc}}{ }^{+}$), implying multi-charge storage ability ${ }^{47}$ for both $1 \mathbf{d}$ and 3 . Compared with 1a, the electron-donating $\mathrm{MeO}$ groups decreased the oxidation potentials by $70 \mathrm{mV}$, while the electron-withdrawing groups increase the oxidation potentials more evidently; for example, $\mathrm{CN}$ groups shifted the oxidation potentials by $\sim 350 \mathrm{mV}$. The oxidation peaks shifted to higher potentials on increasing the number of fluorine atoms $(50-70,250$ and $500 \mathrm{mV}$ for one, two and three fluorine substituents, respectively). Extension of the conjugation of the acene units also decreased the oxidation potentials; for example, to $0.25 \mathrm{~V}$ for 3 with its six conjugated benzene rings and to $0.21 \mathrm{~V}$ for 2 with its eight conjugated benzene rings. Consistent with these findings, 2 and $\mathbf{3}$ appear to be susceptible to slow oxidative degradation in solution; no such degradation appeared in the solutions of the other compounds. All of these compounds exhibited two reduction potentials, with the effects of the substituents on the reduction potentials occurring in the same direction as those on the oxidation potentials, but to a lesser extent. Accordingly, the band gaps of these species were influenced by the substituents and the conjugation length, with electron acceptors decreasing the HOMO energy level and, thereby, increasing the band gap (see Fig. 4f, especially for the trifluorinated derivative).

We performed the DFT calculations at the B3LYP/6-31G(d) level to examine the electronic structures of the dibenzoterryleneacene hybrids. The calculated frontier orbitals indicated that they maintained the characteristics of their individual components. The HOMO and LUMO orbitals of 1a are mainly localized in the central pyrene unit (Supplementary Table 4). We observed nodal planes perpendicular to the molecular skeleton in the HOMOs and LUMOs of these compounds, with the electron densities distributed symmetrically on the two sides of the nodal plane. The HOMO-1 and LUMO +1 orbitals of $\mathbf{1 a}$ are comparable with the HOMO-2 and LUMO +2 orbitals, respectively, of 

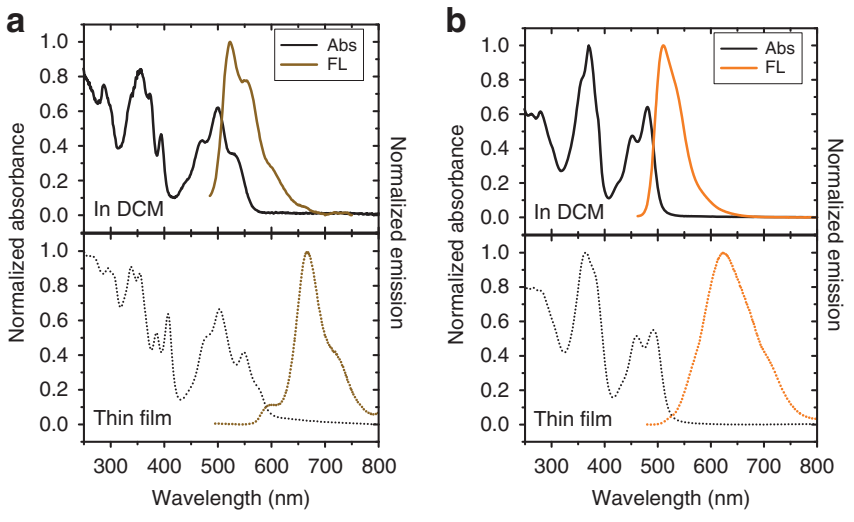

e

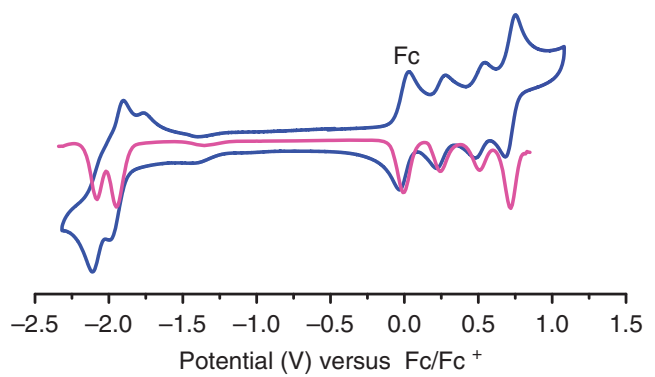

f

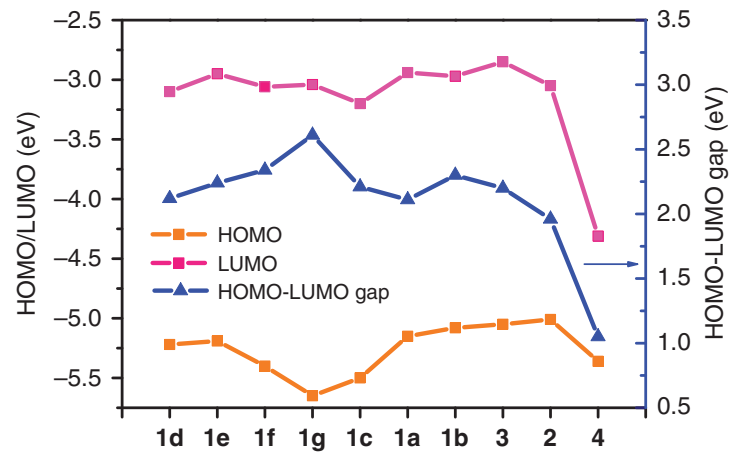

C

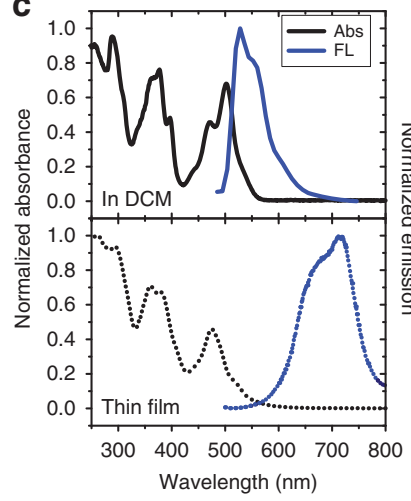

d

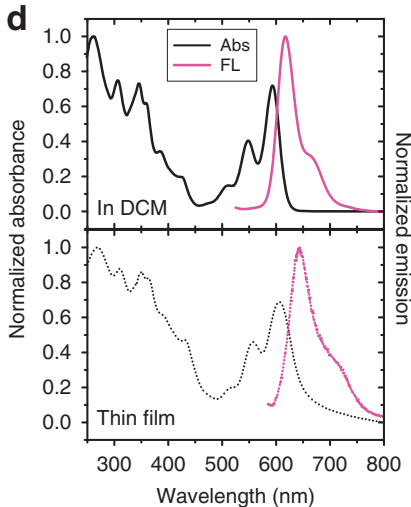

g

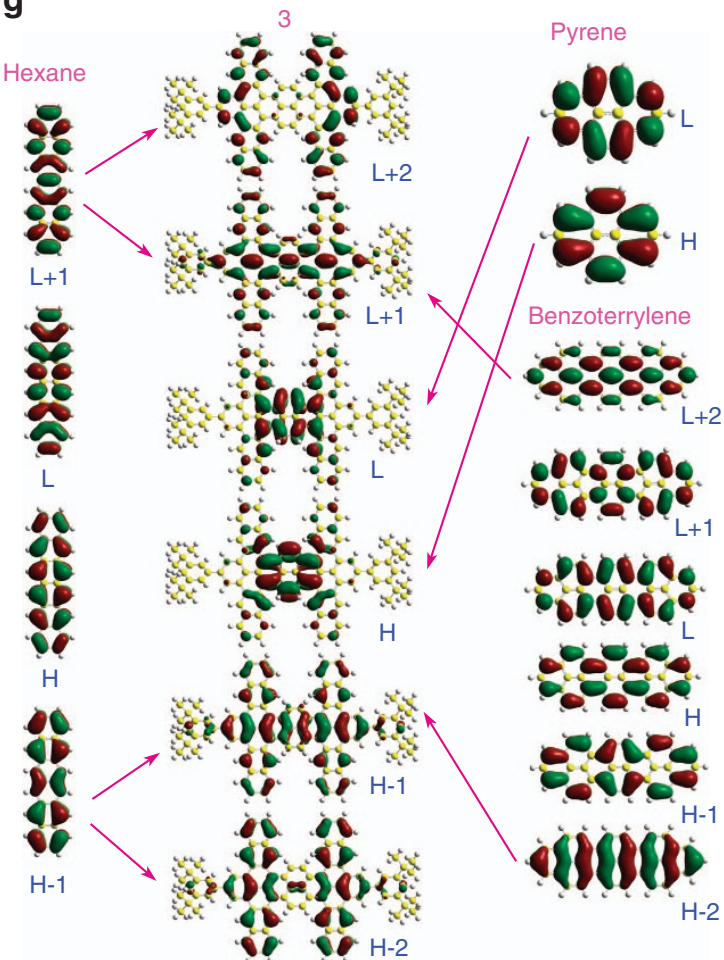

3
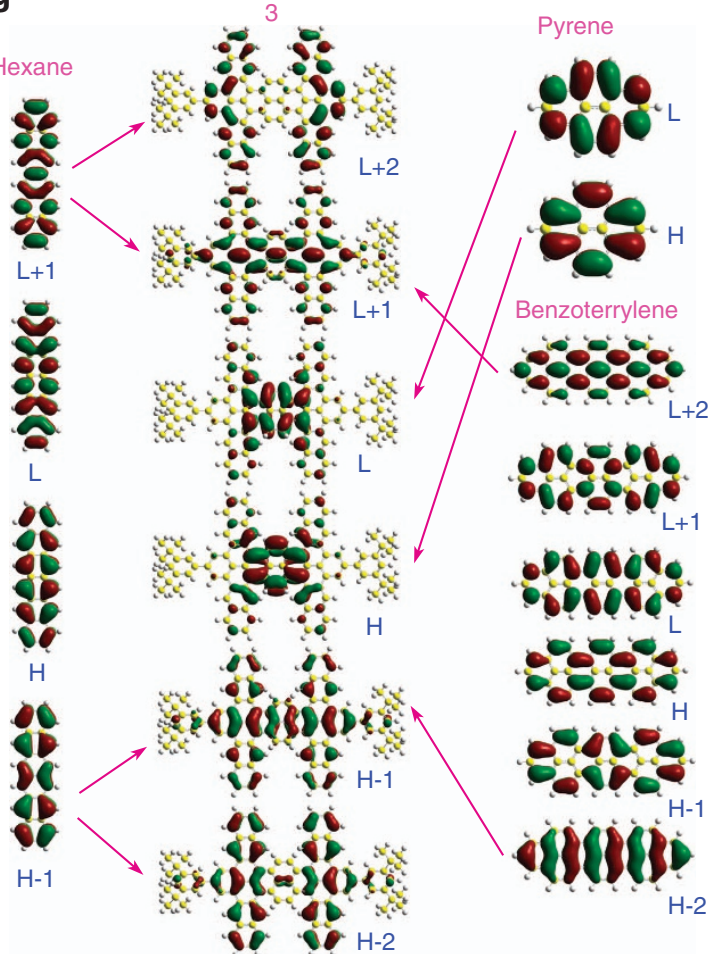

Pyrene

Figure 4 | Optical, electrochemical behaviour and orbital analysis of compounds 1-3. Absorbance (black) and emission (coloured) spectra of (a) 1a, (b) $\mathbf{1 g}$, (c) $\mathbf{1 c}$ and (d) $\mathbf{2}$ recorded in $\mathrm{CH}_{2} \mathrm{Cl}_{2}$ solution (solid lines) and in the solid state (spin-cast from $\mathrm{CH}_{2} \mathrm{Cl}_{2}$ solutions; dotted lines). (e) $\mathrm{CV}$ (blue) and $\mathrm{DPV}$ (red) traces of $\mathbf{3}$ in $\mathrm{CH}_{2} \mathrm{Cl}_{2}(0.1 \mathrm{mM})$ at room temperature. Scan rate: $100 \mathrm{mVs}^{-1}$; working electrode: glassy carbon; reference electrode: Ag wire; electrolyte: $\mathrm{TBAPF}_{6} . \mathrm{Fc}_{\mathrm{Fc}}{ }^{+}$: ferrocene/ferrocenium. (f) $\mathrm{HOMO}$ and LUMO energies and band gaps of 1-4. (g) Frontier orbitals analysis of $\mathbf{3}$ calculated at the B3LYP/6-31G (d) level.

dibenzoterrylene. The other compounds had similarly appearing orbitals, except for 1f, where the strongly electron-withdrawing $\mathrm{CN}$ group affected the polarization of its orbitals (Supplementary Table 4). Interestingly, extension of the acenes vertical to the benzoterrylene, as in $\mathbf{3}$, led to more independent characteristics for the pyrene, benzoterrylene and acene subcomponents (Fig. 4g). Increasing the steric constraint of these acenes led to compounds exhibiting the properties of the central benzoterrylene (for example, for $\mathbf{2}$ in Supplementary Fig. 76). Timedependent DFT calculations revealed that the absorption behaviour of these compounds was the result of a mixture of pyrene-, dibenzoterrylne- and acene-like orbitals (Supplementary Fig. 77, Supplementary Tables 5-11). For 1, the main band in the range $400-600 \mathrm{~nm}$, corresponding to the HOMO-LUMO transition, according to the calculation, was caused by a transition from a pyrene-like HOMO to a pyrene-like LUMO. The second band, near $350 \mathrm{~nm}$, due to the HOMO $-1 \rightarrow \mathrm{LUMO}+1$ transition, was related to the transition between the dibenzoterrylene-like HOMO-2 and LUMO + 2. The small sharp peak near $400 \mathrm{~nm}$ arose from the transition from a pyrene-like HOMO to a dibenzoterrylene-like LUMO +2 . We observed similar transition behaviour for 3, although the band in the $300-400 \mathrm{~nm}$ region was red-shifted by $\sim 50 \mathrm{~nm}$. Consistent with the molecular orbital profiles, 2 displayed a dibenzoterrylene-like HOMO $\rightarrow$ LUMO transition for the main band near $600 \mathrm{~nm}$, with a transition between the dibenzoterrylene-like HOMO-2 and $\mathrm{LUMO}+2$ for the band near $350 \mathrm{~nm}$. We also observed dibenzoterrylene-like $\mathrm{HOMO}-2 \rightarrow \mathrm{LUMO}$ and $\mathrm{HOMO} \rightarrow \mathrm{LUMO}+2$ transitions for the weak band at $344 \mathrm{~nm}$.

Optical and magnetic properties of open-shell compound 4 . Dark-green compound $\mathbf{4}$ provides an absorption spectrum (Fig. 5b) displaying bands with maxima at 834,675 and $585 \mathrm{~nm}$ 
a
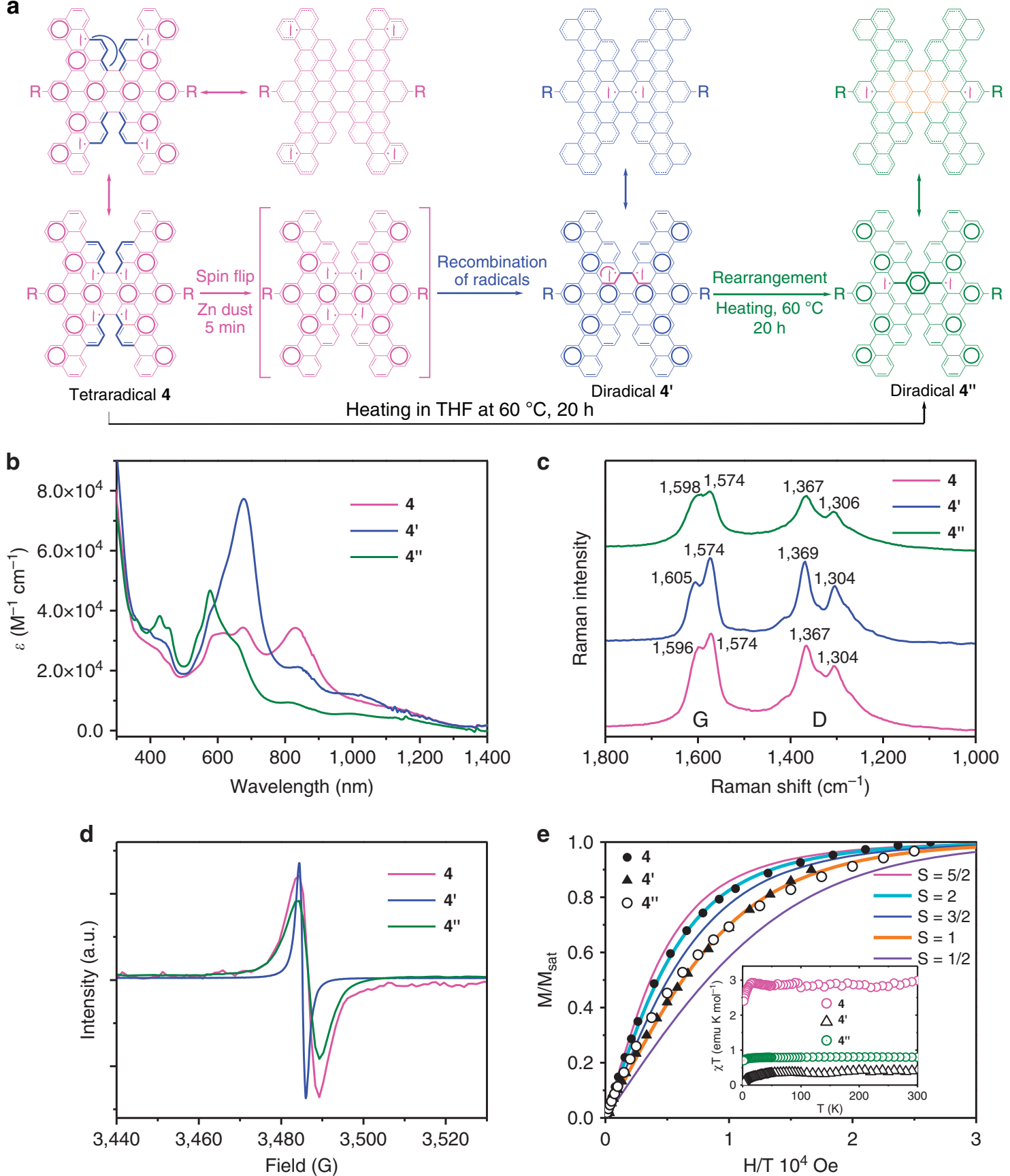

Figure 5 | Spectroscopic characterization of compound 4. (a) Transformation of tetraradical $\mathbf{4}$ to diradicals $\mathbf{4}^{\prime}$ or $\mathbf{4}^{\prime \prime}$ mediated with spin catalysis by Zinc or trace radicals in THF, and the resonance structures of $\mathbf{4}, \mathbf{4}^{\prime}$ and $\mathbf{4}^{\prime \prime}$. (b) UV-vis-near-infrared spectra of $\mathbf{4}, \mathbf{4}^{\prime}$ and $\mathbf{4}^{\prime \prime}$ in $\mathrm{CH}_{2} \mathrm{Cl}_{2}$. (c) Raman spectra of solid 4, 4' and $\mathbf{4}^{\prime \prime}$. (d) ESR spectra of chloroform solutions of $\mathbf{4}, \mathbf{4}^{\prime}$ and $\mathbf{4}^{\prime \prime}$ recorded at room temperature. (e) The magnetization of $\mathbf{4}^{\prime} \mathbf{4}^{\prime}$ and $\mathbf{4}^{\prime \prime}$ are plotted as $\mathrm{M}$ / $\mathrm{M}_{\mathrm{sa}}$ t versus $\mathrm{H} / \mathrm{T}$, with Brillouin functions for $\mathrm{S}=5 / 2,2,3 / 2,1$ and $1 / 2$. The fitting parameters for $\mathbf{4}^{\prime} \mathbf{4}^{\prime}$ and $\mathbf{4}^{\prime \prime}$ are $\mathrm{S}=2.08(1.9 \mathrm{~K}), 1.02(3 \mathrm{~K}), 1.14(1.9 \mathrm{~K})$ and the corresponding $M_{\text {sat }}=0.8,0.37,0.88 \mu \mathrm{B}$, respectively. Inset plots: the magnetic susceptibility of $\mathbf{4}(2,000 \mathrm{Oe}), \mathbf{4}^{\prime}(10,000 \mathrm{Oe})$ and $\mathbf{4}^{\prime \prime}(2,000 \mathrm{Oe})$ are plotted as $\chi \top$ versus $\mathrm{T}$.

with a tail into the near-infrared region (ca. 1,200 nm). We calculated an optically measured band gap of $\sim 1.03 \mathrm{eV}$. The CV trace of 4 (Supplementary Fig. 75) in 1,2-dichlorobenzene $(0.1 \mathrm{M}$ tetrabutylammonium hexafluorophosphate) displayed a one-step irreversible oxidation and a one-step reduction. The HOMO and LUMO energies, calculated through CV, were -5.31 and $-4.26 \mathrm{eV}$, respectively, corresponding to a band gap of $1.05 \mathrm{eV}$, very close to that determined in silico $(1.1 \mathrm{eV})$. In addition, we observed almost no fluorescence from $\mathbf{4}$ in solution and in the solid state.
Raman spectroscopy is a very useful tool for characterizing benzene-type aromatic radicals ${ }^{48}$, because characteristic benzene vibrational Raman bands exist near $1,600 \mathrm{~cm}^{-1}$ that are very sensitive to the electronic configuration within the six-membered benzene ring, either 'benzoquinoidal' or 'benzoaromatic.' The Raman spectrum of $\mathbf{4}$ featured two sets of peaks, much like the D and $\mathrm{G}$ bands of graphene (Fig. $5 \mathrm{c}$ ). The peak near $1,367 \mathrm{~cm}^{-1}$ corresponds to the breathing vibrations of $\mathrm{sp}^{2}$-hybridized carbon domains in aromatic rings (that is, the $\mathrm{D}$ band); we assign the peak near $1,596 \mathrm{~cm}^{-1}$ to the first-order scattering of the $E_{2 \mathrm{~g}}$ 

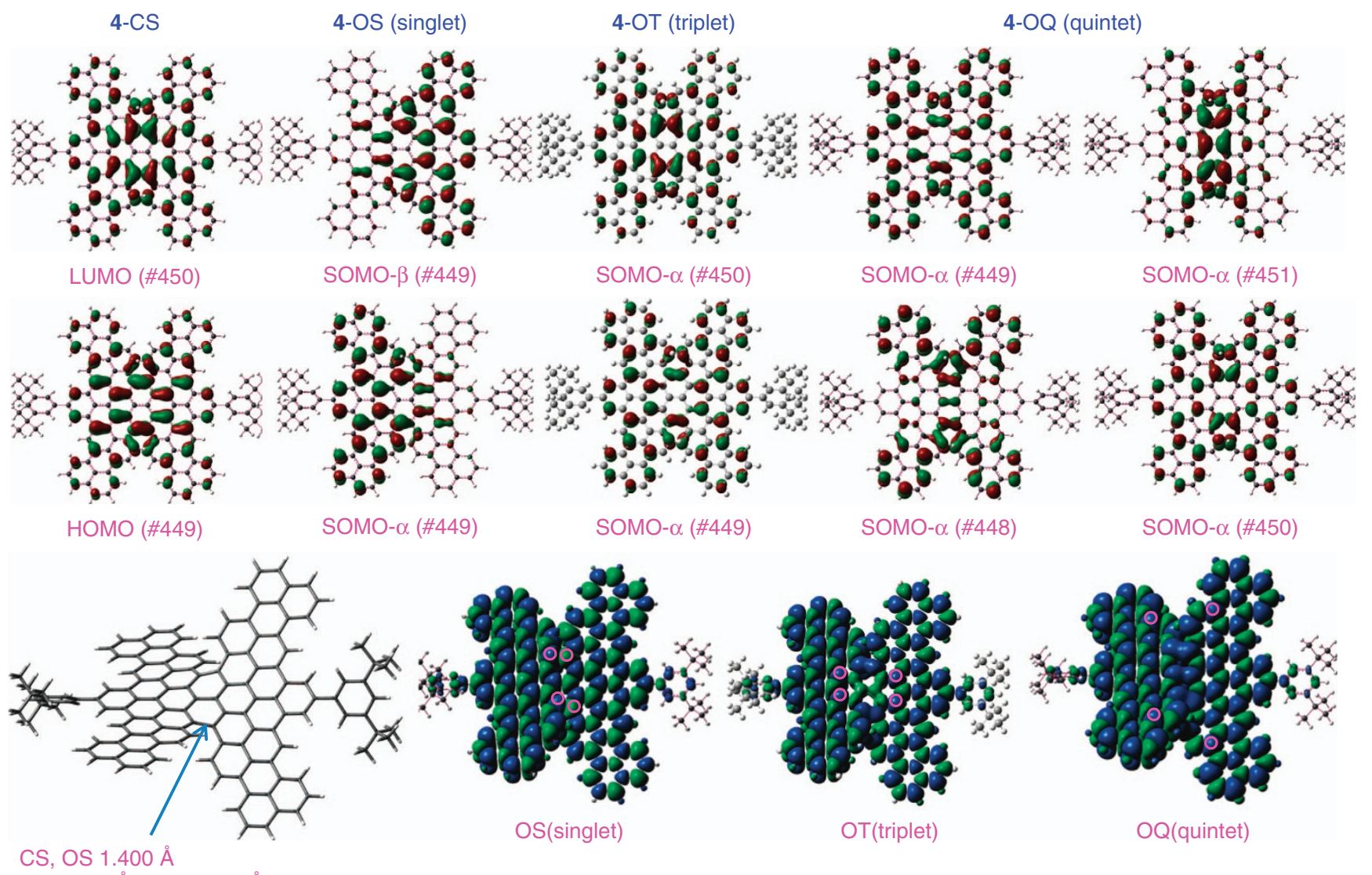

OT $1.401 \AA$ OQ $1.474 \AA$

Figure 6 | Orbitals and spin-density distributions for closed-shell and open-shell compound 4. Calculated (UCAM-B3LYP) HOMOs and LUMOs for 4-CS, SOMOs and spin-density distributions for $\mathbf{4}$ in the singlet $(\mathbf{4}-\mathrm{OS}, \mathrm{S}=0)$, triplet $(\mathbf{4}-\mathrm{OT}, \mathrm{S}=1)$ and quintet state $(\mathbf{4}-\mathrm{OQ}, \mathrm{S}=2)$. Blue and green surfaces represent $\alpha$ - and $\beta$-spin densities, respectively. The red marks indicate the atoms exhibiting highest spin-density. The central bond connecting the two pentacene-annulated-tribenzo-octacene units in quintet-state pocesses the single-bond charateristic, which indicates the less conjugation between the two units than other states.

mode observed for in-phase stretching vibration of $\mathrm{sp}^{2}$-hybridized carbon domains in aromatic rings (that is, the $G$ band) ${ }^{49}$. The downfield-shifting of the $\mathrm{D}$ and $\mathrm{G}$ bands (from 1,367 to $1,304 \mathrm{~cm}^{-1}$ and from 1,596 to $1,574 \mathrm{~cm}^{-1}$, respectively) indicated the coexistence of 'benzoquinoidal' and 'benzoaromatic' benzene rings.

The NMR spectrum of 4 in $\mathrm{C}_{2} \mathrm{D}_{2} \mathrm{Cl}_{4}$ did not feature any signals at room temperature, nor after cooling to $-50{ }^{\circ} \mathrm{C}$, suggesting the presence of a considerable paramagnetic species. In addition, the solution of 4 provided a featureless broad ESR signal (Fig. 5d), resulting from the long-distance spin - spin dipole interaction within the molecules and the extended spin-delocalization ${ }^{48,50,51}$. These data indicate that $\mathbf{4}$ exists as an open-shell multi-radical in the ground state.

We performed SQUID measurements for $\mathbf{4}$ in the powder form as a function of magnetic field $\left(H=0-5 \times 10^{4}\right.$ Oe and $T=1.9$ $\mathrm{K})$ and temperature $(T=2-300 \mathrm{~K}$ at $H=2,000 \mathrm{Oe})$. The value of $S=2.08$, determined from the curvature of the Brillouin plots, indicate the quintet $(S=2)$ ground state for tetraradical 4 (Fig. 5d). The inset of Fig. $5 \mathrm{~d}$ presents a plot of $\chi_{\mathrm{M}} \mathrm{T}(\mathrm{T})$ for 4 . The constant value of $\chi \mathrm{T}\left(2.84 \mathrm{emu} \mathrm{K} \mathrm{mol}^{-1}\right)$, as evidenced by the flatness of the $\chi \mathrm{T}$ versus $\mathrm{T}$ plots in the $\mathrm{T}=30-300 \mathrm{~K}$ range, indicates that there is no significant change in the thermal population of spin states up to $300 \mathrm{~K}$. After removing the temperature independent contribution, the numerical fit of the data by the Currie-Weiss model give Currie constant of $\mathrm{C}=2.84$ emu K mol ${ }^{-1}$ and Weiss constant $\theta_{\mathrm{W}}$ of $-0.38 \mathrm{~K}$ (Supplementary Fig. 78), which indicated weak intermolecular antiferromagnetic interaction below $20 \mathrm{~K}$.
Interestingly, solid 4 displayed high stability, and there was no obvious decomposition when the solid was stored under ambient air and light conditions for months. However, mixing the tetraradical 4 with Zinc dust can generate another radical $\mathbf{4}^{\prime}$, and heating the solution of 4 or $4^{\prime}$ in THF at $60^{\circ} \mathrm{C}$ for about $20 \mathrm{~h}$ will transform both of them to another species $4^{\prime \prime}$ completely. $4^{\prime}$ and $4^{\prime \prime}$ showed no molecular weight change, while the strongest absorption bands of $\mathbf{4}^{\prime}$ and $\mathbf{4}^{\prime \prime}$ were shifted to 678 and $575 \mathrm{~nm}$, respectively, with the absorption band at $834 \mathrm{~nm}$ became weak (Fig. 5b and Supplementary Fig. 81). Similar Raman spectra (Fig. 5c) and magnetic field dependent magnetic signal changes (Fig. 5e) indicate unequivocally that both of $\mathbf{4}^{\prime}$ and $\mathbf{4}^{\prime \prime}$ are triplet biradicals at ground state (Supplementary Figs 79 and 80). The values of $\chi \mathrm{T} \approx 1 \mathrm{emu} \mathrm{K} \mathrm{mol}{ }^{-1}$ were found for $\mathbf{4}^{\prime}$ and $\mathbf{4}^{\prime \prime}$, after values of $\chi \mathrm{T}\left(0.77,0.36 \mathrm{emu} \mathrm{K} \mathrm{mol}^{-1}\right)$ are corrected by spin concentration $\left(M_{\mathrm{sat}}=0.88,0.37 \mu \mathrm{B}\right)$. The transformation from tetraradical to diradicals is probably due to the fact that one pair of electrons in the tetraradical formed one covalent bond by Zinc surface catalysed spin flip ${ }^{52,53}$ (Fig. 5a), the left two electrons delocalized on the two pentacene-annulated-tribenzo-octacene units, respectively, with parallel spin electron patterns at ground state. The obtained diradical $\mathbf{4}^{\prime}$ can be rearranged to a more stable diradical $4^{\prime \prime}$ on heating. Transformation directly from 4 to $4^{\prime \prime}$ in hot THF processed through spin catalysis ${ }^{52,53}$ by the trace radicals in THF, followed by the thermal rearrangement, without the observation of the intermediates. Sharpening ESR signal for $\mathbf{4}^{\prime}$ (Fig. 5d), indicates that the spin - spin dipole interaction distance within the molecules of $\mathbf{4}^{\prime}$ is shorter than that of $\mathbf{4}$ and $\mathbf{4}^{\prime \prime}$. The 
transformation processes and the possible resonance structures of $\mathbf{4}, \mathbf{4}^{\prime}$ and $\mathbf{4}^{\prime \prime}$ are summarized in Fig. 5a, with the supports from the following DFT optimization of the tetraradical and diradicals. Tetraradical 4 can be considered as four phenalenyl radicals annulated by twisted $\mathrm{H}$-shaped acene; intermediate diradical $\mathbf{4}^{\prime}$ featured two close-contacted radical pairs like two over-lapped phenalenyl radicals; $\mathbf{4}^{\prime \prime}$ can be viewed as two phenalenyl radicals fused by central pyrene, with benzene rings surrounded. The detailed spin catalysis mediated transformation mechanism needs further investigation.

Although 1D PAHs (n-acenes) featured the antiferromagnetic ground state due to the zigzag-shaped boundaries, which cause $\pi$-electrons to localize and form spin orders at the edges, the longer acene ground states are polyradical in nature ${ }^{54,55}$. 2D PAHs (periacenes and circumacenes) develops a strong multiradical character with increasing zigzag chain length ${ }^{56,57}$. Our experimental results indicated tetraradical character for the twisted 2D molecule 4 . We performed DFT calculations at the UCAM-B3LYP level of theory to investigate the ground state of the tetraradical 4 (Fig. 6). Because of steric effects, the molecule adopted a twisted planar structure. The structure 4-CS features extended $\pi$-electron delocalization from the pyrene core to the four annulated perylene units, with the central pyrene unit possessing the largest HOMO and LUMO coefficients (Fig. 6), suggesting that it would be the most reactive site. For the broken-symmetry singlet state 4 -OS, we observed disjointed singly occupied molecular orbitals (SOMOs), SOMO- $\alpha$ and SOMO- $\beta$, with orbital coefficients mainly localized at the terminal pentacene-annulated-tribenzo-octacene units. The spindensity distribution reveals delocalization with the central pyrene units having the largest density, especially the K-region carbon atoms (also the $5^{\prime}, 7^{\prime}$ positions of dibenzopentacene) (Supplementary Fig. 82, Supplementary Tables 12-14); this situation is different from that found in bisphenalenyls, where the spin-density is delocalized mainly at the terminal phenalenyl units $^{25}$, with antiparallel spin electron patterns typical for this broken-symmetry singlet state. This $\mathrm{S}=0$ state can be considered as the DBP-4 form in Fig. 1c, which also corresponds to an $S=1$ state when the spin electrons parallel to each other (diradical $\mathbf{4}^{\prime}$ in Fig. 5a). For the high-spin quintet state $(4-O Q, S=2)$, we observed a large spin-density at the 5,10 positions of the octacene core-positions that are blocked by the annulated pentacene, which is the DBO-4 form (Fig. 1c, also the tetraradical 4). However, for the $S=1$ state (4-OT, also the diradical $\mathbf{4}^{\prime \prime}$ in Fig. 5a), the spins are delocalized on the pentacene-annulatedtribenzo-octacene units, with more distribution on the 7,8 positions of the octacene core, well-protected by surrounding benzene rings. From the SOMO- $\alpha$ and SOMO- $\beta$ profiles and the spin-distributions of the singlet 4-OS and high-spin triplet 4-OT and quintet 4-OQ, we ascribe the good chemical stability of the tetraradical toward oxidation and dimerization/oligomerization to its thermodynamic stabilization ${ }^{33}$, resulting from both delocalization and kinetic blocking through benzoannulation.

\section{Discussion}

We have developed an efficient method for the preparation of a series of stable 2D twisted dibenzoterrylene-acenes. We have investigated their electronic structures and geometries in the ground state using various experimental techniques, assisted by DFT calculations. The photophysical, electrochemical, and magnetic properties of these compounds were dependent on the length of the acene. The ground-state structures in this series of molecules were tunable, with 1-3 being closed-shell hydrocarbons and 4 being an open-shell singlet tetraradical. Compound 4 is the first stable twisted 2D hydrocarbon tetraradical obtained by hybridizing dibenzoterrylene with acene, which can be transferred to more thermodynamic stable triplet diradical. The high stability of the tetraradical and diradical arose from thermodynamic stabilization, due to delocalization and kinetic blocking through benzoanullation. We believe that $2 \mathrm{D}$ twisted conjugated molecules with various width and edge structures could also be synthesized in a similar approach. With the installation of different functional groups such as heterocycles on the periphery of such 2D twisted conjugated molecules, specific functionalities on them could be obtained. Combination this approach with polymerization could give great promise for the synthesis of chemically precise, multiple-dimensional polymers. Moreover, the unique optical, electronic, and magnetic properties of these extended 2D acenes suggest that they might be promising candidates for use in nonlinear optics, field effect transistors, and organic spintronics.

\section{Methods}

Materials. Most of the chemical reagents were purchased from Alfa Aesar or Aldrich Chemicals and were utilized as received unless indicated otherwise. All solvents were purified using standard procedures. Column chromatography was performed on silica gel (size 200-300 mesh).

Synthesis procedures of compounds 1-4. For details of the synthetic procedures, see Supplementary Methods. For NMR and high-resolution mass spectra of compounds in this manuscript, see Supplementary Figs 1-68.

Sample characterization. ${ }^{1} \mathrm{H}$ and ${ }^{13} \mathrm{C}$ NMR spectra were recorded on a Bruker AVANCE 400 or Bruker AVANCE III 500WB instrument, at a constant temperature of $25^{\circ} \mathrm{C}$. Chemical shifts are reported in parts per million from low-to-high field and referenced to TMS. Matrix-assisted laser desorption/ionization Fourier transform ion cyclotron resonance (MALDI-FT-ICR-MS) MS were performed on a Bruker Solarix 9.4T FT-ICR-MS mass spectrometer. EI mass spectrometric measurements were performed on a SHIMADZU GCMS-QP2010 puls Spectrometer. Elemental analyses were recorded on a Carlo-Erba-1106 instrument. Electronic absorption spectra were measured on a JASCO V-579 spectrophotometer. Raman spectra were taken on a NT-MDT NTEGRA spectra Raman spectroscopy SPM system. CV experiments were performed using an electrochemical analyser with a conventional three-electrode configuration, glassy carbon electrode as a working electrode and $\mathrm{Ag} / \mathrm{AgCl}$ as a reference electrode. All experiments were performed in $\mathrm{CH}_{2} \mathrm{Cl}_{2}$ with $0.1 \mathrm{M}$ of $n \mathrm{Bu}_{4} \mathrm{NPF}_{6}$ as a supporting electrolyte, the ferrocene/ferrocenium ion $\left(\mathrm{Fc} / \mathrm{Fc}^{+}\right)$as inter-reference. The $\mathrm{E}_{1 / 2}$ values were determined as $1 / 2\left(\mathrm{E}_{\mathrm{pa}}+\mathrm{E}_{\mathrm{pc}}\right)$, where $\mathrm{E}_{\mathrm{pa}}$ and $\mathrm{E}_{\mathrm{pc}}$ are the anodic and cathodic peak potentials, respectively.

X-ray diffraction data analysis. Crystals of 1 e and $\mathbf{2}$ were grown by slow diffusing acetone to the solutions in $\mathrm{CHCl}_{3}$ solution. Single crystal X-ray diffraction data were collected on a Agilent SuperNova (Dual, $\mathrm{Cu}$ at zero, Atlas) diffractometer with $\mathrm{Cu} \mathrm{K} \alpha$ radiation $(\lambda=1.54184 \AA$ ) from micro-focus sealed X-ray tube. Intensities were corrected for absorption effects using the multi-scan technique ${ }^{58}$ The structures were solved by direct methods and refined by a full matrix least squares technique based on $\mathrm{F}^{2}$ using SHELXT program ${ }^{59}$. The refinement details: Refinement of $\mathrm{F}^{2}$ against ALL reflections. The weighted R-factor $\mathrm{wR}$ and goodness of fit $\mathrm{S}$ are based on $\mathrm{F}^{2}$, conventional $\mathrm{R}$-factors $\mathrm{R}$ are based on $\mathrm{F}$, with $\mathrm{F}$ set to zero for negative $\mathrm{F}^{2}$. The threshold expression of $\mathrm{F}^{2}>2$ sigma $\left(\mathrm{F}^{2}\right)$ is used only for calculating R-factors (gt) and so on, and is not relevant to the choice of reflections for refinement. R-factors based on $\mathrm{F}^{2}$ are statistically about twice as large as those based on F, and R-factors based on ALL data will be even larger. For X-ray data see Supplementary Data 1 and 2.

Attempts to refine peaks of residual electron density as solvents led to $12 \mathrm{CHCl}_{3}$ and 6 acetone and some additional peaks of residual electron density which can't be refined successfully. The data were corrected for disordered electron density through use of the SQUEEZE procedure ${ }^{60}$ as implemented in PLATON ${ }^{61}$. A total solvent-accessible void volume of $2,179.1 \mathrm{~A}^{3}$ with a total electron count of 584.3 was found in the unit cell in four voids.

The structure solution of crystal 2 (Cell: 19.2306(4) A, 19.4633(5) $\AA$, 58.6850(14) $\AA, 90^{\circ}$, orthorhombic P c c n, $Z=8$ ) shows two independent half molecules, one of which is disordered with its symmetry equivalent. It is possible that the cell chosen for the current refinement in represents a subcell and that reflections of a larger supercell were too weak to be observed. The current result represents a preliminary connectivity only. We have grown the crystals of this structure several times, the crystals are thin plate, and we tried to collect better data, however, the data reported here is the best we can obtain. The six-membered rings in the phenanthrenyl groups and terminal benzenes with $t$-butyl groups were flattened with AFIX 66 and DFIX. As agreed by the reviewer, although the data is 
poor, it is perfectly fine for the assumptions that there is a twist associated with the molecule as predicted by the DFT calculation. We present with caution some geometrical parameters: phenanthrenyl units twisted with the central naphthene unit in the cis direction.

ESR spectra were obtained with a Bruker E500-10/12 spectrometer. No additional hyperfine coupling was detected at $0.1 \mathrm{G}$, thus experiments were performed at 1.0 or $2 \mathrm{G}$ for improved signal intensities. The sweep width was $83.89 \mathrm{~s}$, time constant was $40.96 \mathrm{~ms}$ and the microwave power was $10.1 \mathrm{~mW}$ (corresponding to attenuation $=13 \mathrm{~dB}$, which was sufficiently high to avoid power saturation).

Magnetic measurements were performed on a Quantum Design MPMS-XL5 SQUID magnetometer. Small amounts of sample material (between 30 and $40 \mathrm{mg}$ ) were put into KLF containers and brought into measuring position using a straw. The variation of the magnetization as a function of temperature at fixed external magnetic field ( $T=2-300 \mathrm{~K}$ at $H=2,000 \mathrm{Oe})$ and as a function of the external field at fixed temperature $\left(H=0-5 \times 10^{4} \mathrm{Oe}\right.$ and $\left.T=1.9 \mathrm{~K}\right)$ was studied. The powder of 4 was sealed in a plastic tube. The signal of sample holder and plastic tube was deducted by measuring the sample holder and plastic tube under same conditions. A correction for the diamagnetism of the sample and the sample container was applied before calculating the susceptibilities from the magnetization data. For all other samples of solid di- and tetraradicals, the correction for diamagnetism was based on high-temperature extrapolation of the $\chi$ versus $1 / T$ plots, that is, a suitable numerical factor $\left(M_{\mathrm{dia}}\right)$ was added to the magnetization $(M)$, until the $\chi T$ versus $T$ plot becomes flat in the high-temperature range.

Theoretical calculations of target compounds 1-4. For compound 1, 2 and 3 , DFT calculations were performed using the Gaussian 03 program $^{62}$, Geometries were optimized in the gas-phase using the B3LYP functional and 6-31 g (d) basis set on all atoms. Theoretical calculations of compound $\mathbf{4}$ were carried out by using the Gaussian 09 suite of programs ${ }^{63}$. The initial geometry optimization of 4 was performed with the UCAM-B3LYP level of theory on the singlet state and the Handy and co-workers ${ }^{66}$ long-range corrected version of B3LYP $6-31 \mathrm{G}^{*}$, and all electron basis sets were used for all atoms ${ }^{64-66}$. The resulting DFT solution (singlet 'closed-shell': zero-spin-density on all atoms) was further tested for its stability with the STABLE $=$ OPT keyword ${ }^{67}$. A spin symmetry broken DFT solution was found with lower energy. Then the Guess = Read keyword was used to perform the optimization at the UCAM-B3LYP level (singlet open-shell). For DFT data see Supplementary Data 3-6.

Data availability. Data referenced in this study are available in 'Cambridge Crystallographic Data Centre (CCDC)' with the accession codes '1457979' and ' 1457980 ', see Supplementary Data 1 and 2. All other data supporting the findings of this study are available within the article and its Supplementary Information files.

\section{References}

1. Fujii, S. \& Enoki, T. Nanographene and graphene edges: electronic structure and nanofabrication. Acc. Chem. Res. 46, 2202-2210 (2013).

2. Yan, X., Cui, X. \& Li, L.-S. Synthesis of large, stable colloidal graphene quantum dots with tunable size. J. Am. Chem. Soc. 132, 5944-5945 (2010).

3. Chen, L., Hernandez, Y., Feng, X. \& Muellen, K. From nanographene and graphene nanoribbons to graphene sheets: chemical synthesis. Angew. Chem. Int. Ed. 51, 7640-7654 (2012).

4. Kawasumi, K. et al. A grossly warped nanographene and the consequences of multiple odd-membered-ring defects. Nat. Chem. 5, 739-744 (2013).

5. Zhang, Q. et al. Facile bottom-up synthesis of coronene-based 3-fold symmetrical and highly substituted nanographenes from simple aromatics. J. Am. Chem. Soc. 136, 5057-5064 (2014).

6. Narita, A. et al. Synthesis of structurally well-defined and liquid-phaseprocessable graphene nanoribbons. Nat. Chem. 6, 126-132 (2014).

7. Cai, J. et al. Atomically precise bottom-up fabrication of graphene nanoribbons. Nature 466, 470-473 (2010).

8. Treier, M. et al. Surface-assisted cyclodehydrogenation provides a synthetic route towards easily processable and chemically tailored nanographenes. Nat. Chem. 3, 61-67 (2011).

9. Vo, T. H. et al. Large-scale solution synthesis of narrow graphene nanoribbons. Nat. Commun. 5, 3189 (2014).

10. Ozaki, K. et al. One-shot K-region-selective annulative pi-extension for nanographene synthesis and functionalization. Nat. Commun. 6, 6251 (2015).

11. Schuler, B. et al. From perylene to a 22-ring aromatic hydrocarbon in one-pot. Angew. Chem. Int. Ed. 53, 9004-9006 (2014).

12. Kastler, M. et al. From armchair to zigzag peripheries in nanographenes. J. Am. Chem. Soc. 128, 9526-9534 (2006).

13. Wang, Z. H. et al. Graphitic molecules with partial 'Zig/Zag' periphery. J. Am. Chem. Soc. 126, 7794-7795 (2004).

14. Ritter, K. A. \& Lyding, J. W. The influence of edge structure on the electronic properties of graphene quantum dots and nanoribbons. Nat. Mater. 8, 235-242 (2009).
15. Zhang, L. et al. Unconventional, chemically stable, and soluble twodimensional angular polycyclic aromatic hydrocarbons: from molecular design to device applications. Acc. Chem. Res. 48, 500-509 (2015).

16. Anthony, J. E. The larger acenes: versatile organic semiconductors. Angew. Chem. Int. Ed. 47, 452-483 (2008).

17. Chun, D., Cheng, Y. \& Wudl, F. The most stable and fully characterized functionalized heptacene. Angew. Chem. Int. Ed. 47, 8380-8385 (2008).

18. Purushothaman, B. et al. Synthesis and structural characterization of crystalline nonacenes. Angew. Chem. Int. Ed. 50, 7013-7017 (2011).

19. Watanabe, M. et al. The synthesis, crystal structure and charge-transport properties of hexacene. Nat. Chem. 4, 574-578 (2012).

20. Lambert, C. Towards polycyclic aromatic hydrocarbons with a singlet openshell ground state. Angew. Chem. Int. Ed. 50, 1756-1758 (2011).

21. Morita, Y., Suzuki, S., Sato, K. \& Takui, T. Synthetic organic spin chemistry for structurally well-defined open-shell graphene fragments. Nat. Chem. 3, 197-204 (2011).

22. Sun, Z., Ye, Q., Chi, C. \& Wu, J. Low band gap polycyclic hydrocarbons: from closed-shell near infrared dyes and semiconductors to open-shell radicals. Chem. Soc. Rev. 41, 7857-7889 (2012).

23. Abe, M. Diradicals. Chem. Rev. 113, 7011-7088 (2013),

24. Kamada, K. et al. Strong two-photon absorption of singlet diradical hydrocarbons. Angew. Chem. Int. Ed. 46, 3544-3546 (2007).

25. Kubo, T. et al. Synthesis, intermolecular interaction, and semiconductive behavior of a delocalized singlet biradical hydrocarbon. Angew. Chem. Int. Ed. 44, 6564-6568 (2005).

26. Morita, Y. et al. Organic tailored batteries materials using stable open-shell molecules with degenerate frontier orbitals. Nat. Mater. 10, 947-951 (2011).

27. Anthony, J. E. Functionalized acenes and heteroacenes for organic electronics. Chem. Rev. 106, 5028-5048 (2006).

28. Zade, S. S. \& Bendikov, M. Heptacene and beyond: the longest characterized acenes. Angew. Chem. Int. Ed. 49, 4012-4015 (2010).

29. Einholz, R. \& Bettinger, H. F. Heptacene: increased persistence of a $4 \mathrm{n}+2 \pi$-electron polycyclic aromatic hydrocarbon by oxidation to the $4 \mathrm{n} \pi$-electron dication. Angew. Chem. Int. Ed. 52, 9818-9820 (2013).

30. Zade, S. S. et al. Products and Mechanism of acene dimerization. a computational study. J. Am. Chem. Soc. 133, 10803-10816 (2011).

31. Kaur, I. et al. Design, synthesis, and characterization of a persistent nonacene derivative. J. Am. Chem. Soc. 132, 1261-1263 (2010).

32. Pérez, D., Peña, D. \& Guitián, E. Aryne cycloaddition reactions in the synthesis of large polycyclic aromatic compounds. Eur. J. Org. Chem. 2013, 5981-6013 (2013).

33. Rath, H. et al. A stable organic radical delocalized on a highly twisted pi system formed upon palladium metalation of a mobius aromatic hexaphyrin. Angew. Chem. Int. Ed. 49, 1489-1491 (2010).

34. Wu, J. S. et al. Controlled self-assembly of hexa-peri-hexabenzocoronenes in solution. J. Am. Chem. Soc. 126, 11311-11321 (2004).

35. Pisula, W., Feng, X. \& Muellen, K. Tuning the columnar organization of discotic polycyclic aromatic hydrocarbons. Adv. Mater. 22, 3634-3649 (2010).

36. Chen, L. et al. Hexathienocoronenes: synthesis and self-organization. J. Am. Chem. Soc. 134, 17869-17872 (2012).

37. Diez-Perez, I. et al. Gate-controlled electron transport in coronenes as a bottom-up approach towards graphene transistors. Nat. Commun. 1, 31 (2010).

38. Yamamoto, Y. et al. Photoconductive coaxial nanotubes of molecularly connected electron donor and acceptor layers. Science 314, 1761-1764 (2006).

39. Feng, X. et al. Controlled self-assembly of C-3-symmetric hexa-perihexabenzocoronenes with alternating hydrophilic and hydrophobic substituents in solution, in the bulk, and on a surface. J. Am. Chem. Soc. 131, 4439-4448 (2009).

40. Hill, J. P. et al. Self-assembled hexa-peri-hexabenzocoronene graphitic nanotube. Science 304, 1481-1483 (2004).

41. Kastler, M. et al. Influence of alkyl substituents on the solution- and surface-organization of hexa-peri-hexabenzocoronenes. J. Am. Chem. Soc. 127, 4286-4296 (2005).

42. Xiao, S. et al. Controlled doping in thin-film transistors of large contorted aromatic compounds. Angew. Chem. Int. Ed. 52, 4558-4562 (2013).

43. Cohen, Y. S. et al. Enforced one-dimensional photoconductivity in corecladding hexabenzocoronenes. Nano Lett. 6, 2838-2841 (2006).

44. Kang, S. J. et al. A supramolecular complex in small-molecule solar cells based on contorted aromatic molecules. Angew. Chem. Int. Ed. 51, 8594-8597 (2012).

45. Marsden, J. A. \& Haley, M. M. Carbon networks based on dehydrobenzoannulenes. 5. extension of two-dimensional conjugation in graphdiyne nanoarchitectures. J. Org. Chem. 70, 10213-10226 (2005).

46. Luo, J., Xu, X., Mao, R. \& Miao, Q. Curved polycyclic aromatic molecules that are pi-isoelectronic to hexabenzocoronene. J. Am. Chem. Soc. 134, 13796-13803 (2012).

47. Echegoyen, L. \& Herranz, M. A. Fullerene Electrochemistry. Fullerenes: From Synthesis To Optoelectronic Properties 4, 267-293 (2002).

48. Li, Y. et al. Kinetically blocked stable heptazethrene and octazethrene: closed-shell or open-shell in the ground state? J. Am. Chem. Soc. 134, 14913-14922 (2012) 
49. Tuinstra, F. \& Koenig, J. L. Raman spectrum of graphite. J. Chem. Phys. 53, $1126(1970)$

50. Shimizu, A. et al. Alternating covalent bonding interactions in a one-dimensional chain of a phenalenyl-based singlet biradical molecule having Kekulé structures. J. Am. Chem. Soc. 132, 14421-14428 (2010).

51. Sun, Z., Huang, K. \& Wu, J. Soluble and stable heptazethrenebis(dicarboximide) with a singlet open-shell ground state. J. Am. Chem. Soc. 133, 11896-11899 (2011).

52. Buchachenko, A. L. \& Berdinsky, V. L. Electron spin catalysis. Chem. Rev. 102, 603-612 (2002).

53. Khavryuchenko, O. V., Khavryuchenko, V. D. \& Su, D. S. Spin catalysts: a quantum trigger for chemical reactions. Chinese J. Catal. 36, 1656-1661 (2015).

54. Jiang, D.-E., Sumpter, B. G. \& Dai, S. First principles study of magnetism in nanographenes. J. Chem. Phys. 127, 124703 (2007).

55. Hachmann, J., Dorando, J. J., Avilés, M. \& Chan, G. K.-L. The radical character of the acenes: a density matrix renormalization group study. J. Chem. Phys. 127, 134309 (2007)

56. Jiang, D. E. \& Dai, S. Electronic ground state of higher acenes. J. Phys. Chem. A 112, 332-335 (2008).

57. Plasser, F. et al. The Multiradical character of one- and two-dimensional graphene nanoribbons. Angew. Chem. Int. Ed. 52, 2581-2584 (2013)

58. CrysAlisPro 1.171.38.41; Agilent Technologies Ltd.: Yarton, Oxfordshire, UK, 2015.

59. Sheldrick, G. M. SHELXT- integrated space-group and crystal-structure determination. Acta Cryst. A71, 3-8 (2015).

60. van der Sluis, P. \& Spek, A. L. BYPASS: an effective method for the refinement of crystal structures containing disordered solvent regions. Acta Cryst A 46, 194-201 (1990)

61. Spek, A. L. PLATON-a multipurpose crystallographic tool. Acta Cryst. Vol. A 46, C34 (Utrecht University 1990).

62. Frisch, M. J. et al. Gaussian 03, Revision B (Gaussian, Inc., 2003).

63. Frisch, M. J. et al. Gaussian09, Revision E.01 (Gaussian, Inc., 2009).

64. Lee, C., Yang, W. \& Parr, R. G. Development of the Colle-Salvetti correlationenergy formula into a functional of the electron density. Phys. Rev. B: Condens. Matter Mater. Phys. 37, 785-789 (1988).

65. Becke, A. D. Density-functional thermochemistry. III. The role of exact exchange. J. Chem. Phys. 98, 5648-5652 (1993).

66. Yanai, T., Tew, D. \& Handy, N. A new hybrid exchange - correlation functional using the coulomb-attenuating method (CAM-B3LYP). Chem. Phys. Lett. 393, 51-57 (2004).
67. Seeger, R. Pople, self-consistent molecular orbital methods. XVIII. constraints and stability in Hartree-Fock theory. J. Chem. Phys. 66, 3045-3050 (1977).

\section{Acknowledgements}

This work was supported by the National Natural Science Foundation of China (21290190, 21322301, 91227113), NSFC-DFG joint fund (21261130581), the National Basic Research 973 Program of China (2012CB932901), and the 'Strategic Priority Research Program' of the Chinese Academy of Sciences (XDA09020302, XDB12010300).

\section{Author contributions}

Yongjun Li and Yuliang Li conceived and designed the project and co-wrote the manuscript, with assistance from the other authors. Yongjun Li and Z.J. prepared the materials, Yongjun Li performed the computation, the ESR and SQUID data were analysed by S.X. and H.L., respectively. All authors discussed the results and contributed to manuscript preparation.

\section{Additional information}

Supplementary Information accompanies this paper at http://www.nature.com/ naturecommunications

Competing financial interests: The authors declare no competing financial interests.

Reprints and permission information is available online at http://npg.nature.com/ reprintsandpermissions/

How to cite this article: Li, Y. et al. A method for controlling the synthesis of stable twisted two-dimensional conjugated molecules. Nat. Commun. 7:11637 doi: 10.1038/ ncomms11637 (2016)

(c) (i) This work is licensed under a Creative Commons Attribution 4.0 International License. The images or other third party material in this article are included in the article's Creative Commons license, unless indicated otherwise in the credit line; if the material is not included under the Creative Commons license, users will need to obtain permission from the license holder to reproduce the material. To view a copy of this license, visit http://creativecommons.org/licenses/by/4.0/ 